\title{
Multi-functional star tracker - future perspectives
}

\author{
J. Roháč, M. Řeřábek, R. Hudec
}

\begin{abstract}
This paper focuses on the idea of a multi-functional wide-field star tracker (WFST) and provides a description of the current state-of-the-art in this field. The idea comes from a proposal handed in to ESA at the beginning of 2011. Star trackers (STs) usually have more than one object-lens with a small Field-of-View. They provide very precise information about the attitude in space according to consecutive evaluation of star positions. Our idea of WFST will combine the functions of several instruments, e.g. ST, a horizon sensor, and an all-sky photometry camera. WFST will use a fish-eye lens. There is no comparable product on the present-day market. Nowadays, spacecraft have to carry several instruments for these applications. This increases the weight of the instrumentation and reduces the weight available for the payload.
\end{abstract}

Keywords: star tracker, horizon sensor, all-sky camera, photometry, astrometry, space variant image processing.

\section{Introduction}

Attitude determination functions are basic functions in all space applications. Attitude information is used in Attitude and Orbit Control Systems to stabilize a spacecraft $(\mathrm{S} / \mathrm{C})$ in the required position and orientation. Various attitude reference sources can be used for obtaining attitude information, e.g. Earth horizon sensors, which can be directed towards the Sun, Moon, or other planets and stars, Sun sensors, magnetometers, and star trackers (STs). However, only STs provide accuracy of attitude estimation better than 30 arcseconds [1]. The first generation of STs acquired only a few bright stars in the Fieldof-View (FoV) and provided the focal plane coordinates of these stars [2]. The coordinates were not related to inertial space, and the attitude information therefore had to be provided indirectly by an external unit. This was due the insufficient power of the microcomputers. Rapid improvements in microcomputer power led to the autonomous functionality of STs which became the preferred attitude reference source. Basically, the second generation ST consists of an electronic camera and a microcomputer as shown in Figure 1. The ST autonomously performs pattern recognition of stars in FoV, compares the results with the star catalogue stored in the internal memory, and estimates the attitude. Generally, the $\mathrm{ST}$ has to operate in two modes. The first mode solves the lost-in-space function which does not have previous attitude information available, and therefore the star pattern in the FoV has to be recognized. The identification can be usually accomplished in a few seconds. The other mode performs star tracking, which assumes that the current attitude is closely related to the previous attitude. This mode is easier for computation because it only tracks previously identi- fied stars at known positions. The basic drivers of ST designs take into account: accuracy, reliability, mass, power consumption, and size. Typical requirements are stated in Tab.1. There are also other parameters that influence the design. They are: star light sensitivity, the detection threshold, the average number of stars in the FoV, and sky coverage.

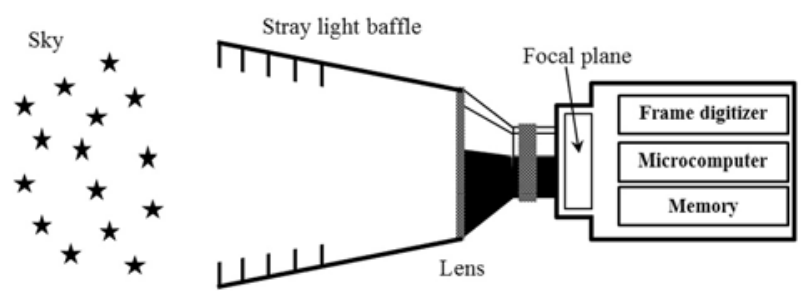

Fig. 1: Principle sketch of a second generation star tracker

Generally, second generation STs employ more than one Camera Head Unit (CHU) consisting of the baffle, the lens, and the optical sensor. It usually has about $20^{\circ} \times 20^{\circ}$ FoV The multi-CHU concept increases the average number of stars in the FoV and the accuracy of the ST. In addition, due to this concept full immunity against simultaneous blinding is ensured

\section{New concept of a multi-functional star tracker}

Second generation STs already have better parameters than required, see Table 1 . Nevertheless, they use the multi-camera-head concept to increase the average of stars in the FoV. To decrease the mass and size of the current ST concept and to increase its functionality we proposed to design, develop, and verify the multi-functional wide-field star tracker 
Table 1: Typical requirements for autonomous star trackers and an example of the Micro Advanced Stellar Compass ( $\mu$ ASC) flown on the PROBA-2 technology demonstration satellite

\begin{tabular}{|l|l|l|}
\hline & Required & $\mu \mathrm{ASC}$ \\
\hline Initial acquisition (lost-in-space function) & $<1 \mathrm{~min}$. & $30 \mathrm{msec}$ \\
\hline Accuracy $(\mathrm{EOL})(\mathrm{arcsec})$ & $30(3 \sigma)$ & $2(3 \sigma)$ \\
\hline Attitude rate $(\mathrm{deg} / \mathrm{sec})$ & up to 1 & up to 20 \\
\hline Availability $(\%)$ & 99.900 & 99.995 \\
\hline Power $(\mathrm{W})$ & $<10$ & $<4$ \\
\hline Mass $(\mathrm{kg})$ & $<2$ & $<0.5$ \\
\hline
\end{tabular}

(WFST), which combines the functions of several instruments usually used in S/Cs.

One function of the WFST will be to provide precise attitude determination. This will be ensured by sophisticated algorithms using the widest possible FoV optic characteristics, supported by innovative image and data processing, a 3D Inertial Measurement Unit (IMU) consisting of accelerometers and angular rate sensors. For low orbit applications, the system will also be equipped with a GPS receiver. All available sources of information, see Figure 2, will be fused by the Extended Kalman Filter (EKF). A two-speed updating approach, as proposed in [3], will be used for mechanizing and computing the inertial navigation equations. The precision will be between 1 arcminute and 1 arcsecond, according the application and the optics that are used.
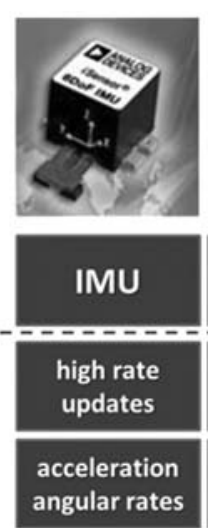
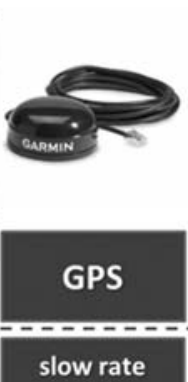

updates

position
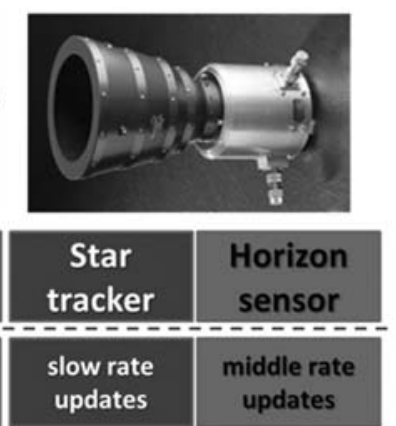

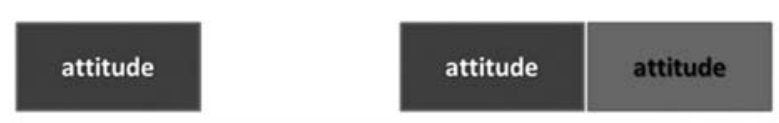

Data fusion by Extended Kalman Filter ATTITUDE ESTIMATOR

Fig. 2: Available data sources for attitude evaluation

The second function focuses on having costeffective means to provide a high-potential information source for scientific analyses of objects in the FoV of the WFST, which extends the applicability of WFST. This idea corresponds fully with ESA Cos- mic vision 2015-2025, where the priorities are planet and Universe studies. WFST will be capable of monitoring fluctuations in the radiation of stars. High potential will be provided by the fish-eye lens, which will enable observations of up to $180 \mathrm{deg}$ sky, and by an innovative approach to image processing. Systems of this kind using a fish-eye lens are commonly referred to as Ultra Wide Field Camera (UWFC) systems. UWFC image data analysis is very difficult in general, due to the wide range of distortions in wide field images and mainly due to the spatial variant (SV) properties of UWFC systems. Moreover, the objects in ultra-wide field images are very small (a few pixels per stellar object).

Precise astronomical measurements (in astrometry and photometry) need high quality images. Even small distortions may lead to inaccurate determination of the position or movement of stellar objects. The error depends on the magnitude of the measured star - the higher it is, the higher the error can be. The properties of UWFC astronomical systems and the specific visual data in astronomical images lead to complicated evaluation of the image data. These UWFC systems contain many different kinds of optical aberrations that have negative impacts on the image quality and system transfer characteristics. For precise astrometry and photometry over the entire FoV it is therefore very important to comprehend how the optical aberrations affect the transfer characteristics, how the astronomical measurements depend on the optical aberrations, and how the wavefront aberration affects the point spread function (PSF).

Precise stellar profile fitting is very important for astronomical measurements. Nowadays, there are two common functions for fitting stellar profiles, referred to as Gaussian and Moffat functions [4,5]. Efforts are being made to match a star's profile with the Gaussian or Moffat profile and to remember the parameters of the fit. If a star were ideal, the stellar profile would be represented by a small "dot". Due to many different distortions, the dot is blurred all around. The more it is blurred, the worse is the PSF of the whole imaging system. The centre of a star 
usually has a profile corresponding to the Gaussian function. The more distant parts of the star are, the closer the profile will be to the Moffat function. Therefore, the ideal fitting function should combine these two profiles. The properties of the UWFC system are referred to as space or spatial variant properties, and the PSF of this system is different for each point in the object plane. In our case, a sophisticated algorithm employing special functions based on Zernike polynomials has to be applied in order to get a precise stellar profile fit for the entire FoV.

\section{Conclusion}

The research and development of the multi-functional WFST will verify current technology potentials based on analyses reflecting fast current technological development and improvements of digital optical sensors (CMOS, CCD), low-cost IMU consisting of MEMS (Micro-Electro-Mechanical-System) sensors, and GPS technology. All analyses will determine the boundaries in the instrument functionality with respect to $\mathrm{S} / \mathrm{C}$ maneuvering parameters, various optical sensor sizes, sensitivity, error sources, stability, lifetime, power consumption, mass, and suitability of various wide FoV optics. The proposed WFST should use cost-effective components and thus fill a gap in the market with a system that is novel and powerful, but cost-effective.

\section{Acknowledgement}

This work has been performed under SCIEXNMSch - CHVI and GA 205/09/1302 "Study of sporadic meteors and weak meteor showers using automatic video intensifier cameras" of the Grant Agency of the Czech Republic.

\section{References}

[1] Jørgensen, J. L., Pickles, A.: Fast and robust pointing and tracking using a second generation star tracker, Proceedings of SPIE. Washington : 1998, p. 51-61.

[2] Liebe, C. Ch.: Accuracy Performance of Star Trackers - A Tutorial, IEEE Transactions on Aerospace and Electronic Systems, Vol. 38, No. 2, 2002, p. 587-599.

[3] Savage, P. G.: Strapdown analytics. Strapdown Associates, Minnesota, USA : 2000.

[4] Rerabek, M., Pata, P., Koten, P.: Processing of the Astronomical Image Data obtained from UWFC Optical Systems, Proceedings of SPIE, Washington : 2008.

[5] Starck, J., Murtagh, F.: Astronomical Image and Data Analysis. Berlin : Springer, 2002.

\section{Jan Roháč}

Faculty of Electrical Engineering

Czech Technical University in Prague

Technická 2, 16627 Prague, Czech Republic

Martin Řeřábek

Faculty of Electrical Engineering

Czech Technical University in Prague

Technická 2, 16627 Prague, Czech Republic

Multimedia Signal Processing Group

Ecole Polytechnique Fédérale de Lausanne

Station 11, Lausanne, Switzerland

René Hudec

Faculty of Electrical Engineering

Czech Technical University in Prague

Technická 2, 16627 Prague, Czech Republic 